\title{
Distress, uncertainty, and positive experiences associated with receiving information on personal genomic risk of melanoma
}

\author{
Amelia K Smit $\mathbb{D}^{1,2,7} \cdot$ Ainsley J Newson $\mathbb{D}^{2} \cdot$ Megan Best $\mathbb{C}^{2,3} \cdot$ Caro-Anne Badcock ${ }^{1} \cdot$ Phyllis N Butow ${ }^{4} \cdot$ Judy Kirk $^{5} \cdot$ \\ Kate Dunlop ${ }^{6} \cdot$ Georgina Fenton ${ }^{1} \cdot$ Anne E Cust $\mathbb{D}^{1,7}$
}

Received: 11 September 2017 / Revised: 6 March 2018 / Accepted: 20 March 2018 / Published online: 30 April 2018

(c) European Society of Human Genetics 2018

\begin{abstract}
The aim of this research was to understand how genomics-based personal melanoma risk information impacts psychological and emotional health outcomes in the general population. In a pilot randomized controlled trial, participants $(n=103)$ completed the Multidimensional Impact of Cancer Risk Assessment (MICRA) questionnaire, 3 months after receiving personal melanoma genomic risk information. Mean scores for MICRA items and subscales were stratified by genomic risk group (low, average, high), gender, education, age, and family history of melanoma. $P$ values were obtained from $t$-tests and analysis of variance tests. We found that overall, participants (mean age: 53 years, range: $21-69 ; 52 \%$ female) had a total MICRA mean score of 18.6 (standard deviation: 11.1, range: 1-70; possible range: 0-105). The high genomic risk group had higher mean scores for the total $\left(24.2, \mathrm{~F}_{2,100}=6.7, P=0.0019\right)$, distress $\left(3.3, \mathrm{~F}_{2,100}=9.4, P=0.0002\right)$ and uncertainty $(8.5$, $\left.\mathrm{F}_{2,100}=6.5, P=0.0021\right)$ subscales compared with average (17.6, 1.1, and 4.5 , respectively) and low-risk groups (14.1, 0.5 , and 2.5, respectively). Positive experiences scores were consistent across risk groups. In conclusion, MICRA scores for the total, distress and uncertainty subscales in our study were relatively low overall, but people who receive a high genomic risk result may benefit from increased support following testing.
\end{abstract}

Electronic supplementary material The online version of this article (https://doi.org/10.1038/s41431-018-0145-z) contains supplementary material, which is available to authorized users.

Amelia K Smit

amelia.smit@sydney.edu.au

1 Cancer Epidemiology and Prevention Research, Sydney School of Public Health, The University of Sydney, Sydney, Australia

2 Sydney Health Ethics, Sydney School of Public Health, The University of Sydney, Sydney, Australia

3 Psycho-Oncology Co-operative Research Group (PoCoG), The University of Sydney, Sydney, Australia

4 Centre for Medical Psychology and Evidence-based Decisionmaking, School of Psychology, The University of Sydney, Sydney, Australia

5 Westmead Clinical School, and Westmead Institute for Medical Research, Sydney Medical School, The University of Sydney, Sydney, Australia

6 The Centre for Genetics Education, NSW Health, Sydney, Australia

7 Melanoma Institute Australia, The University of Sydney, Sydney, Australia

\section{Introduction}

The acceleration of genomic technologies has led to improved understanding of the contribution of common genomic variants in common, complex diseases [1]. Genomic risk can represent a wide distribution of diseaserisk probabilities in the general population, and could be incorporated into population-based risk stratification and personalized prevention and screening strategies [2]. However, translation into routine clinical practice requires further research on the broader impact of genomic risk information on psychological and emotional health outcomes in the general population, to help identify individuals who may benefit from additional support from health professionals. Emotions may also influence individuals' processing of health information and engagement in healthy behaviors [3]; thus, understanding psychological impacts of genomic risk information could provide greater insight into any behavior change or lack thereof [4].

Previous research, including in the context of direct-toconsumer testing, has not revealed adverse psychological effects of receiving genomic risk information $[5,6]$; however, many studies have had a high risk of bias [7] and the 
generalizability of their findings to the general population is unclear. Few studies have measured psychological outcomes in individuals unselected by family history of disease using measures, which are specific to genetic testing, such as the Multidimensional Impact of Cancer Risk Assessment (MICRA) [8] instrument. The MICRA has been used predominately in the context of breast cancer patients receiving BRCA1/2 genetic testing results to identify subgroups of vulnerable patients by assessing distress, uncertainty, and positive experiences related to genetic testing. The MICRA instrument has been found to be more sensitive to the specific experiences related to genetic testing and cancer risk compared with general psychological measures, which tend to not detect distress in this context [9]. More recently, the MICRA has also been used in some studies involving healthy individuals receiving genome sequencing results for a range of health and non-health-related conditions $[10,11]$. However, it is unknown whether the MICRA instrument can identify vulnerable subgroups in the general population receiving genomic (polygenic) risk information.

In our pilot randomized controlled trial (RCT) [12], participants without a previous melanoma and unselected for family disease history completed the MICRA instrument 3 months after receiving information on their personal genomic risk of melanoma. Our study found that this intervention was acceptable and feasible and there was some evidence of improved sun protection behaviors [12].

This paper presents our planned analysis of results from the MICRA instrument. We hypothesized that MICRA scores may differ according to participants' genomic risk level. Our novel findings are important for understanding the impact of personal genomic risk information on psychological outcomes in the general population, and for assessing the sensitivity of the MICRA instrument for identifying vulnerable individuals who receive genomic risk information outside the high-risk mutation context.

\section{Materials and Methods}

\section{Study design and methods}

The study design, methods, and main behavioral results have been reported previously [12]. In brief, participants were recruited from a research database managed by the Cancer Council New South Wales, Australia. This database comprises people from the general population, as well as cancer patients and their family and friends who volunteered and gave consent to be contacted about participating in ethically approved cancer research. In this study, the consent rate was $41 \%$ and 118 participants completed a baseline questionnaire and gave a saliva sample. No participants had a personal history of melanoma, and 30 (29\%) reported a family history of melanoma although participants were not selected for their family history. All participants were offered personal melanoma genomic risk information together with a generic educational booklet that contained information on melanoma prevention and early detection behaviors.

The personal risk information was presented in a hardcopy booklet and was sent by mail with the educational booklet. Prior to receiving the booklet, all participants received a telephone call from a genetic counselor, which was guided by a detailed manual developed by the research team [13]. In brief, the call involved an explanation of the personal risk information and verbal probes to check participant understanding and to provide an opportunity to ask questions or raise concerns. Participants randomized to the intervention arm received their risk information on average 3.5 months (range: 1.8-4.9 months) after providing a saliva sample, whereas those in the waitlist control arm received their risk information at the end of the main study, on average 7 months (range: 6.1-10.5 months) after providing their saliva sample.

Participants completed a questionnaire 3 months after receiving their personal risk information, which, in addition to the MICRA, included measures of behaviors (such as sun protection, self-reported sun exposure), mediators of behavior change (such as perceived risk of melanoma, control over developing future melanomas), and psychosocial outcomes (such as skin cancer related worry).

The melanoma risk estimates were based on 42 common genetic variants (single-nucleotide polymorphisms, SNPs) in 21 genes associated with melanoma risk; the list is published in a previous paper [12]. All variants had either a confirmed association with melanoma risk in adequately powered and replicated genome-wide association studies ( $p$ values $<5 \times 10^{-8}$ ) [14] or via whole-genome sequencing approaches (MITF rs149617956 variant) [15]. Participants were presented with their polygenic risk estimates in written and graphical formats, showing their relative risk, their remaining lifetime (absolute) risk, a comparative remaining lifetime risk for an individual of the same gender and age residing in New South Wales, and their risk category (low, average or high genomic risk; Fig. 1). The presentation and delivery of the personal genomic risk information was informed by both our focus group research [16, 17] and relevant disease-risk communication literature [18, 19]. The risk categories were based on quartile cut-points of genomic risk within each age and sex strata, for which participants were classified as low (bottom 25\%), average (middle 50\%), or high (top 25\%) genomic risk. In our sample, the remaining lifetime risks ranged from 0.2 to $9.3 \%$ (median $2.3 \%$ ) for women and from 0.6 to $19.5 \%$ (median $3.9 \%$ ) for men. Relative risk estimates ranged from 0.1 to 3.1 (median 
0.81 , mean $=1$ ) for women and from 0.1 to 4.4 (median 0.85 , mean $=1$ ) for men.

\section{MICRA}

The 3-month questionnaire included the MICRA [8], a validated instrument that measures the impact of receiving cancer genetic testing results; elevated scores indicate greater vulnerability to genetic testing related distress. There were 25 questions (items), and participants rated the frequency of each item on a four-point Likert scale $(0=$ Never, $1=$ Rarely, $3=$ Sometimes, 5= Often) (Supplementary Table 1). We replaced the wording in the original instrument, "test result", with "risk information" to make the items specific to the information delivered in our study.

The total MICRA score was based on a mean score of 21 items, including three subscales that were also analyzed separately: distress (six items), uncertainty (nine items), and positive experiences (four items). The positive experiences subscale was reverse scored, thus higher scores indicate lower positive experiences. The total score also includes an individual item specific to understanding choices for prevention and early detection, and an item specific to feeling regret since receiving the risk information. The instrument also includes a subscale (two items) regarding worry about children (rated only by participants who have children); and a subscale (two items) regarding coping with current or previous cancer diagnosis (for those who have had cancer).

\section{Statistical analysis}

Mean scores were calculated for the total MICRA score and each subscale. We compared the means for the total score and each subscale according to genomic risk category (low, average, high risk), gender, education (high school or equivalent, certificate/diploma/university degree), age (18-44 years, 45-69 years), family history of melanoma (yes, no), and personal or family history of other cancer (yes, no). The categorization of ages was selected a priori based on a commonly used cut-point for stratifying age into younger and older age groups [20]. Education cut-points were based on people with tertiary education likely having better health literacy [21]. Differences between groups were compared using $t$-tests and analysis of variance tests. A multivariate model examining correlates of a high total MICRA score (using the median 17 as a cut-point for low/ high) was constructed with all covariates listed above, using logistic regression with SAS software to generate odds ratios (OR) and 95\% confidence intervals (CI). Statistical significance was inferred at $P<0.05$; no adjustments were made for multiple comparisons because this was a pre-
Table 1 Characteristics of participants who completed 3-month follow-up

\begin{tabular}{lllll}
\hline Characteristics & $\begin{array}{l}\text { Overall, } \\
n(\%)\end{array}$ & $\begin{array}{l}\text { Participants, } n(\%) \text { according to } \\
\text { genomic risk group }\end{array}$ \\
\cline { 3 - 5 } & & $\begin{array}{l}\text { Low-risk } \\
(n=25)\end{array}$ & $\begin{array}{l}\text { Average- } \\
\text { risk }(n=49)\end{array}$ & $\begin{array}{l}\text { High-risk } \\
(n=29)\end{array}$ \\
\hline Age group & & & & \\
$\begin{array}{l}\text { 18-44 years } \\
\text { 45-69 years }\end{array}$ & $28(27)$ & $7(28)$ & $12(24)$ & $9(31)$ \\
$\begin{array}{l}\text { Gender } \\
\begin{array}{l}\text { Female } \\
\text { Male }\end{array}\end{array}$ & $54(52)$ & $15(72)$ & $37(76)$ & $20(69)$ \\
$\begin{array}{l}\text { Education } \\
\begin{array}{l}\text { High school or } \\
\text { equivalent }\end{array}\end{array}$ & $22(21)$ & $5(20)$ & $12(24)$ & $5(17)$ \\
$\begin{array}{l}\text { Certificate/diploma/ } \\
\text { university degree }\end{array}$ & $81(79)$ & $20(80)$ & $37(76)$ & $24(83)$ \\
$\begin{array}{l}\text { Family history of } \\
\text { melanoma }\end{array}$ & $30(29)$ & $8(32)$ & $14(29)$ & $8(28)$ \\
$\begin{array}{l}\text { Personal or family } \\
\text { history of cancer }\end{array}$ & $48(47)$ & $8(32)$ & $26(53)$ & $14(48)$ \\
\begin{tabular}{l} 
Has children \\
\hline
\end{tabular} & $71(69)$ & $18(72)$ & $34(69)$ & $19(66)$ \\
\hline
\end{tabular}

planned analysis. In addition, MICRA scores were not the primary outcome for the pilot trial so the study was not powered to find significant subgroup differences across genomic risk groups.

\section{Results}

\section{Participants}

A total of $103(87 \%)$ participants completed the MICRA instrument. The mean age of participants was 53 years (range: 21-69) and 52\% were female (Table 1).

\section{Total MICRA score}

The mean total score was 18.6 (standard deviation (SD) 11.1; range 0-70) out of a possible maximum score of 105 . This differed across genomic risk categories, with a higher score for the high genomic risk group $\left(\mathrm{F}_{2,100}=6.68, P=\right.$ 0.0019; mean scores shown in Table 2). In a multivariate model containing gender, age category, education level, genomic risk category and family history of melanoma, only genomic risk (low, average, high) was statistically significantly associated with a total MICRA score above the median: OR 6.5 (95\% CI 1.8-23.0) for high-risk and OR 3.2 (95\% CI 1.1-9.7) for average-risk, compared to the lowrisk group $\left(\chi^{2}=8.4, P=0.02\right)$. 
Table 2 MICRA instrument scores, overall and stratified by genomic risk category

\begin{tabular}{|c|c|c|c|c|c|c|c|}
\hline Items (possible range) & Number of items & $\begin{array}{l}\text { Overall } \\
(n=103) \\
\mathrm{M}(\mathrm{SD})\end{array}$ & $\begin{array}{l}\text { Low-risk } \\
(n=25) \\
\mathrm{M}(\mathrm{SD})\end{array}$ & $\begin{array}{l}\text { Average-risk } \\
(n=49) \\
\mathrm{M}(\mathrm{SD})\end{array}$ & $\begin{array}{l}\text { High-risk } \\
(n=29) \\
\text { M (SD) }\end{array}$ & $\mathrm{F}_{2,100}$ & $P$ value $^{\mathrm{a}}$ \\
\hline Total score $(0-105)$ & 21 & $18.6(11.1)$ & $14.1(8)$ & $17.6(8.1)$ & $24.2(15.2)$ & 6.68 & 0.0019 \\
\hline Distress subscale $(0-30)$ & 6 & $1.6(2.9)$ & $0.5(1.2)$ & $1.1(2.3)$ & $3.3(3.9)$ & 9.42 & 0.0002 \\
\hline Uncertainty subscale $(0-45)$ & 9 & $5.1(6.7)$ & $2.5(4.4)$ & $4.5(5.1)$ & $8.5(9.0)$ & 6.54 & 0.0021 \\
\hline $\begin{array}{l}\text { Positive experiences subscale } \\
(0-20)\end{array}$ & 4 & $8.2(5.5)$ & $6.8(6.0)$ & $8.3(5.6)$ & $9.2(4.8)$ & 1.28 & 0.28 \\
\hline Understanding choices item $(0-5)$ & 1 & $2.7(2.1)$ & $3.3(2.0)$ & $2.7(2.1)$ & $2.1(1.9)$ & 2.35 & 0.10 \\
\hline $\begin{array}{l}\text { Worry about children subscale } \\
(0-10)\end{array}$ & 2 & $3.4(2.9)$ & $2.9(2.3)$ & $2.9(2.7)$ & $4.7(3.4)$ & 2.87 & 0.063 \\
\hline $\begin{array}{l}\text { Worry about cancer subscale } \\
(0-10)\end{array}$ & 2 & $3.0(2.0)$ & $4(1.8)$ & $2.5(1.8)$ & $3.1(2.0)$ & 2.44 & 0.099 \\
\hline
\end{tabular}

${ }^{\mathrm{a}}$ Comparing mean scores across genomic risk categories

${ }^{\mathrm{b}}$ Only participants with children responded to these items

${ }^{c}$ Only participants with a personal history of cancer responded to these items

There was no significant difference in the total score by age group (18-44 vs. 45-69 years) overall, but when examined within genomic risk categories, total MICRA scores differed between age groups $\left(\mathrm{F}_{2,97}=3.85, P=0.02\right)$; the largest difference was observed in the high genomic risk group with younger participants having a higher score but this was not statistically significant (mean difference 7.4; SD 4.1, $\mathrm{t}_{97}=-1.79, P=0.08$ ).

\section{Distress subscale}

The mean distress score was 1.6 (SD 2.9; range 0-19), which differed according to genomic risk category, with a higher score for the high-risk group $\left(\mathrm{F}_{2,100}=9.42\right.$, $P=0.0002$; Table 2). Overall distress scores did not significantly differ by the other covariate subgroups, but when examined within genomic risk categories, distress scores were not consistent across age groups $\left(\mathrm{F}_{2,97}=4.75\right.$, $P=0.01)$ and family history of melanoma $\left(\mathrm{F}_{2,84}=3.42\right.$ $P=0.04$ ) (Supplementary Figure 1a). Among those within a high-risk category, higher mean distress scores were observed for younger participants (mean difference 2.7 comparing those aged 18-44 years vs aged $45-69$ years, $\left.\mathrm{t}_{97}=-2.63, P=0.004\right)$, and participants with a family history of melanoma (mean difference 3.1 compared with those without a family history, $\mathrm{t}_{84}=2.91$, $P=0.005)$.

\section{Uncertainty subscale}

The mean uncertainty score was 5.1 (SD 6.7; range 0-39), and this differed according to genomic risk category $\left(\mathrm{F}_{2,100}\right.$ $=6.54, P=0.0021$; Table 2). Overall uncertainty scores did not significantly differ by other covariate subgroups, but when examined within genomic risk categories, the uncertainty scores were not consistent across age group $\left(\mathrm{F}_{2,97}=\right.$ $3.10, P=0.05$ ) or according to family history of melanoma, although the differences were not statistically significant $\left(F_{2,84}=1.94, P=0.15\right)$ (Supplementary Figure $\left.1 b\right)$. Among participants within the high-risk category, those with a family history of melanoma had higher mean uncertainty scores (mean difference 5.3 compared to those without a family history, $\mathrm{t}_{84}=2.0, P=0.05$ ).

\section{Positive experiences subscale}

The positive experiences subscale had an overall mean of 8.2 (SD 5.5; range 0-20), and there were no significant differences between genomic risk categories (Table 2) or when further stratified by the other covariates (Supplementary Figure 1c).

\section{Regret item}

Overall, 98 (95\%) of participants reported 'never' feeling regret about receiving their risk information.

\section{Understanding choices item}

There were no meaningful differences in scores regarding understanding choices for prevention and early detection when stratified by genomic risk category (Table 2) or the other covariates.

\section{Worry about children subscale}

For scores regarding worry about children, subgroup analysis showed that overall men had higher mean worry about 
a

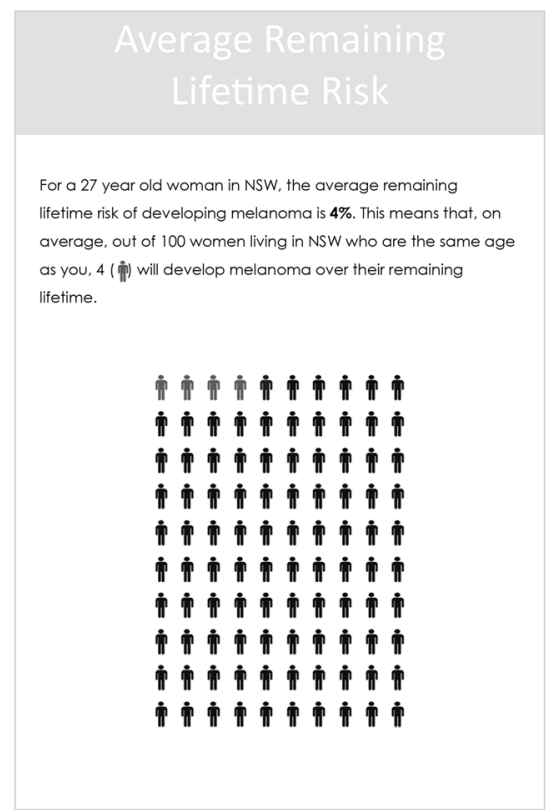

b

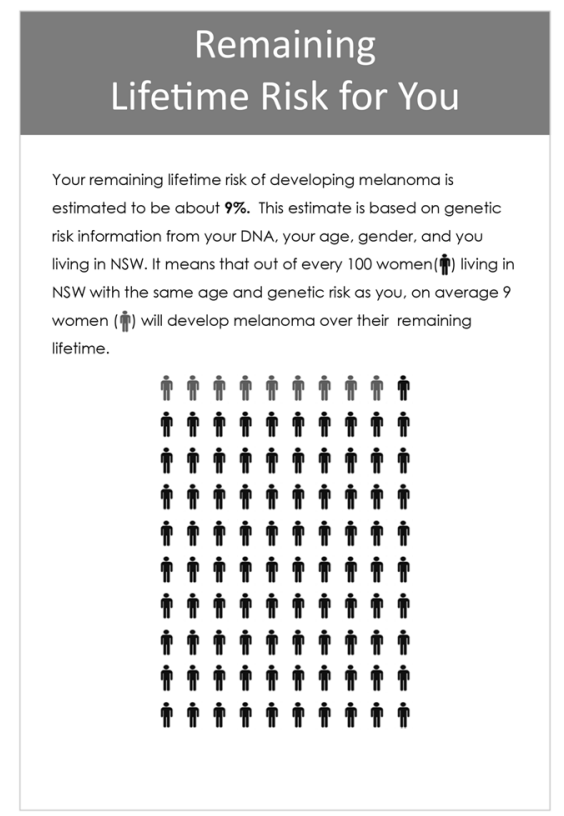

C

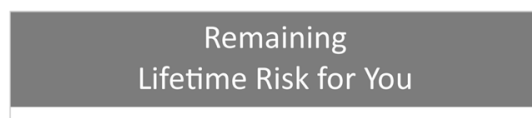

Based on your genetic risk, you are about 2.4 times more at risk of developing melanoma from now until the age of 85 , than other women your age in NSW.

This places you in a high risk category. However, this does not take into account non-genetic risk factors such as past sunburns (see page 9).

Everyone is at risk of developing melanoma. Your risk estimate does not mean that you will develop melanoma even if you are at high risk. If your risk estimate is low you could still develop melanoma.

Being aware of your risk is important, since it's the first step in taking precautions to reduce your risk.

Fig. 1 This is an example of the risk information provided to all participants in the pilot trial in the hard-copy booklet. a The average remaining lifetime (absolute) risk for a person of the same age and gender as the participant and residing in NSW. b The remaining lifetime (absolute) risk for the participant. c The participant's relative risk and genomic risk category

children scores than women (mean 4.1 vs $2.7, \mathrm{t}_{70}=-2.03$, $P=0.046)$.

\section{Worry about cancer subscale}

There were no important differences in scores regarding worry about cancer when stratified by genomic risk category (Table 2) or the other covariates.

\section{Discussion}

Two key findings from our study are that the MICRA scores for distress and uncertainty were relatively low compared with those previously reported $[8,22]$, and that the mean total, distress and uncertainty scores differed significantly according to genomic risk category, with the high-risk group reporting the highest mean MICRA scores.

Most other studies that have used the MICRA have involved delivering genetic testing results for higher penetrance genes (such as $B R C A 1 / 2$ ) to participants selected on the basis of personal or family disease history $[8,22]$. The relatively low overall scores in our study are consistent with our expectation that the emotional and psychological impact of delivering melanoma genomic risk information to the general population would be lower than for delivering genetic testing results for high penetrance genes in families with a relevant disease history. Melanoma is also highly preventable through modifying sun-related behaviors, which do not require rigorous lifestyle changes. This may lead to the perception that melanoma is less threatening than other cancers and therefore may elicit different psychological responses. Educational information emphasizing that melanoma is preventable was provided to all participants in our study.

In addition, disease-risk information based on multiple common genomic variants (i.e., polygenic risk) is based on gene variants with low-moderate penetrance and has less direct implications for family members. Other studies of general psychological responses to receiving results for genetic susceptibility to common health conditions have found minimal psychological reactions in people without a relevant disease history [23]. Nevertheless, we did find some differences in distress and uncertainty scores for participants in the high-risk group with a family history of melanoma. This aligns with our previous finding that people perceive their personal genomic risk of melanoma information as 'shared' with family members [24].

Some studies have involved healthy participants who elected to undergo genetic testing. In a study by Lewis et al., 29 healthy participants in the ClinSeq study who had received clinical genome and exome sequencing results for a range of health conditions (not limited to cancer) had mean distress (1.7, SD 3.9) and uncertainty scores (4.3, SD 7.0) [10] that were comparable to the mean subscale scores in our study (1.6 and 5.1, respectively). Notably, the mean score for positive experiences was relatively high in Lewis et al.'s study (mean 15.2, SD 5.6), indicating fewer positive 
experiences as this subscale is reverse scored. The mean score for positive experiences in our study (mean 8.2, SD 5.5 ) is similar to a study by Sanderson et al., who reported a mean score of 7.45 (SD: 6.83) for 29 participants (healthy individuals who took part in the HealthSeq study) 6-months after they received health and non-health-related wholegenome sequencing results [11]. A novel aspect of our study is that we compared MICRA scores across different genomic risk groups, whereas the ClinSeq and HealthSeq studies reported overall scores.

At 6-months follow-up Sanderson et al. found that the mean distress score was 0.48 (SD 1.27), which they described as lower than the score at 1-week follow-up (mean 1.69, SD 4.0); these authors did not use the uncertainty subscale. Distress and concerns related to genetic testing results have also been found to decrease over time by other studies $[11,25]$. In our study, participants completed the MICRA 3 months after receiving their risk information; this time-point was chosen to measure shortterm behavioral outcomes and may have influenced our findings. There is not a recommended time-point for using the MICRA although outcomes might be most salient in the first few weeks after receipt of genetic test findings. Delivery of the MICRA in other studies ranges from 1 week to 4 years $[10,25]$. Future studies that measure MICRA scores at multiple time-points during follow-up will help to understand how support needs may change over time.

The higher distress and uncertainty scores among participants at higher melanoma risk compared with those at average or low-risk in our study were consistent with studies by Cella et al. [8] and Bjornslett et al. [22], which found that recipients of BRCA1/2-positive results had significantly higher total MICRA scores. Our positive experiences scores did not vary according to any covariates. Conversely, other studies have reported higher positive experience scores (i.e., worse positive experiences) for participants at higher disease risk $[8,22]$. In addition, very few participants reported feeling regret about receiving their risk information in our study. Consistent positive experiences and few participants feeling regret are noteworthy findings because positive responses can influence how an individual processes health information and this may impact their health-related behaviors [4].

We previously reported finding no differences in general psychological distress between people who received (intervention arm), or did not receive (waitlist control arm), their risk information, using the five-item version of the Mental Health Inventory [26] that measured general psychological distress and well-being [12]. Other studies have similarly found that participants with higher genetic risk have higher MICRA scores but that this difference was not reflected in more general measures of distress [8, 22]. In the findings presented here, which includes all participants from both study arms after they received their genomic risk information, we found evidence that the MICRA instrument is more sensitive to measuring the specific impact of result disclosure after genetic testing than other general measures of distress and may be more appropriate for this context.

The MICRA is a validated scale and has been widely used in research studies to measure the specific types of worry and distress relevant to receiving genetic testing results, which are not captured in general measures of distress [9]. A limitation of the MICRA is that there are no widely accepted clinical cut-points [8]. Previous studies have suggested that patients with "elevated" MICRA scores (defined as significantly higher mean scores) may be more vulnerable to distress related to genetic testing and cancer risk evaluation $[8,22]$. Genetic testing related distress and uncertainty could have implications for factors such as healthcare-related decision making, including screening [25], however, further research is required.

In our study, the significantly higher distress (mean: 3.3, SD: 3.9) and uncertainty (mean: 8.5, SD: 9.0) scores in people with a high genomic risk of melanoma are an indication that they could be more susceptible to genetic testing related distress. Further research is needed to identify the clinical implications of higher distress and uncertainty scores, in addition to the type of support that may be beneficial and the clinical cut-points at which people may require increased support.

Participants in our study were recruited from a cancer research database, and this may limit the generalizability of our findings to the broader population. In addition, the use of the term "high risk" in our study may have influenced participant responses and future research could explore how different risk descriptions might impact participant outcomes especially when absolute risk remains relatively low. The polygenic risk score was based on the best available evidence, and validation of this risk score is ongoing. Although ours is one of the largest studies using the MICRA in a general population sample, we had limited power to evaluate differences by genomic risk subgroup and by demographic characteristics within each risk category. Therefore, our results based on these stratified results should be considered preliminary and require a larger study to confirm our findings.

In conclusion, our preliminary findings indicate that delivery of personal genomic risk of melanoma to the general population is unlikely to elicit high levels of distress or uncertainty. However, people who receive a high genomic risk result may have elevated levels of uncertainty and distress and could potentially benefit from increased genetic counseling support. Recipients of this information had positive experiences regardless of their genomic risk category, which may have beneficial implications for preventive behaviors. 
Acknowledgements This study received funding from Sydney Catalyst Translational Cancer Research Centre and The University of Sydney Cancer Strategic Priority Area for Research Collaboration (SPARC) Implementation Scheme. AE Cust received Career Development Fellowships from the National Health and Medical Research Council of Australia (NHMRC; 1147843) and Cancer Institute NSW (15/CDF/1-14). AK Smit received a Research Training Program (RTP) Stipend Scholarship and a Merit Top Up Scholarship from the University of Sydney, and a Top Up Scholar Award from the Sydney Catalyst Translational Cancer Research Centre.

\section{Compliance with ethical standards}

Conflict of interest The authors declare that they have no conflict of interest.

\section{References}

1. Green ED, Guyer MS, National Human Genome Research I. Charting a course for genomic medicine from base pairs to bedside. Nature. 2011;470:204-13.

2. Burton H, Chowdhury S, Dent T, Hall A, Pashayan N, Pharoah P. Public health implications from COGS and potential for risk stratification and screening. Nat Genet. 2013;45: 349-51.

3. Kiviniemi MT, Voss-Humke AM, Seifert AL. How do I feel about the behavior? The interplay of affective associations with behaviors and cognitive beliefs as influences on physical activity behavior. Health Psychol. 2007;26:152-8.

4. O'Neill SC, Tercyak KP, Baytop C, Hensley Alford S, McBride CM. A new approach to assessing affect and the emotional implications of personal genomic testing for common disease risk. Public Health Genom. 2015;18:104-12.

5. Bloss CS, Wineinger NE, Darst BF, Schork NJ, Topol EJ. Impact of direct-to-consumer genomic testing at long term follow-up. J Med Genet. 2013;50:393-400.

6. Godino JG, van Sluijs EM, Marteau TM, Sutton S, Sharp SJ, Griffin SJ. Lifestyle advice combined with personalized estimates of genetic or phenotypic risk of type 2 diabetes, and objectively measured physical activity: a randomized controlled trial. PLoS Med. 2016;13:e1002185.

7. Hollands GJ, French DP, Griffin SJ, et al. The impact of communicating genetic risks of disease on risk-reducing health behaviour: systematic review with meta-analysis. BMJ. 2016;352: i1102.

8. Cella D, Hughes C, Peterman A, et al. A brief assessment of concerns associated with genetic testing for cancer: the Multidimensional Impact of Cancer Risk Assessment (MICRA) questionnaire. Health Psychol. 2002;21:564-72.

9. Graves KD, Vegella P, Poggi EA, et al. Long-term psychosocial outcomes of BRCA1/BRCA2 testing: differences across affected status and risk-reducing surgery choice. Cancer Epidemiol Biomark Prev. 2012;21:445-55.

10. Lewis KL, Hooker GW, Connors PD, et al. Participant use and communication of findings from exome sequencing: a mixedmethods study. Genet Med. 2016;18:577-83.
11. Sanderson SC, Linderman MD, Suckiel SA, et al. Psychological and behavioural impact of returning personal results from wholegenome sequencing: the HealthSeq project. Eur J Hum Genet. 2017;25:280-92.

12. Smit AK, Espinoza D, Newson AJ, et al. A pilot randomized controlled trial of the feasibility, acceptability, and impact of giving information on personalized genomic risk of melanoma to the public. Cancer Epidemiol Biomark Prev. 2017;26:212-21.

13. Fenton GL, Smit AK, Freeman L. et al. Development and evaluation of a telephone communication protocol for the delivery of personalized melanoma genomic risk to the general population. J. Genet. Couns. 2017; https://doi.org/10.1007/ s10897-017-0183-7.

14. Law MH, Bishop DT, Lee JE, et al. Genome-wide meta-analysis identifies five new susceptibility loci for cutaneous malignant melanoma. Nat Genet. 2015;47:987-95.

15. Yokoyama S, Woods SL, Boyle GM, et al. A novel recurrent mutation in MITF predisposes to familial and sporadic melanoma. Nature. 2011;480:99-103.

16. Smit AK, Keogh LA, Hersch J, et al. Public preferences for communicating personal genomic risk information: a focus group study. Health Expect. 2016;19:1203-14.

17. Smit AK, Keogh LA, Newson AJ, Hersch J, Butow P, Cust AE. Exploring the potential emotional and behavioural impact of providing personalised genomic risk information to the public: a focus group study. Public Health Genom. 2015;18:309-17.

18. Hawley ST, Zikmund-Fisher B, Ubel P, Jancovic A, Lucas T, Fagerlin A. The impact of the format of graphical presentation on health-related knowledge and treatment choices. Patient Educ Couns. 2008;73:448-55.

19. Lautenbach DM, Christensen KD, Sparks JA, Green RC. Communicating genetic risk information for common disorders in the era of genomic medicine. Annu Rev Genom Hum Genet. 2013;14:491-513.

20. 45 And Up Study C, Banks E, Redman S, et al. Cohort profile: the 45 and up study. Int J Epidemiol. 2008;37:941-7.

21. van der Heide I, Wang J, Droomers M, Spreeuwenberg $P$, Rademakers J, Uiters E. The relationship between health, education, and health literacy: results from the Dutch Adult Literacy and Life Skills Survey. J Health Commun. 2013;18:172-84.

22. Bjornslett M, Dahl AA, Sorebo O, Dorum A. Psychological distress related to BRCA testing in ovarian cancer patients. Fam Cancer. 2015;14:495-504.

23. Kaphingst KA, McBride CM, Wade C, et al. Patients' understanding of and responses to multiplex genetic susceptibility test results. Genet Med. 2012;14:681-7.

24. Smit AK, Keogh LA, Newson AJ et al. Does personalized melanoma genomic risk information trigger conversations about skin cancer prevention and skin examination with family, friends and health professionals? Br J Dermatol 2017;177:779-790.

25. Halbert CH, Stopfer JE, McDonald J, et al. Long-term reactions to genetic testing for BRCA1 and BRCA2 mutations: does time heal women's concerns? J Clin Oncol. 2011;29:4302-6.

26. Berwick DM, Murphy JM, Goldman PA, Ware JE Jr, Barsky AJ, Weinstein MC. Performance of a five-item mental health screening test. Med Care. 1991;29:169-76. 